\title{
Generalized Green-Kubo formulas for fluids with impulsive, dissipative, stochastic, and conservative interactions
}

\author{
M. H. Ernst ${ }^{1,2}$ and R. Brito ${ }^{2,3}$ \\ ${ }^{1}$ Institute for Theoretical Physics, Universiteit Utrecht, 3508 TD Utrecht, The Netherlands \\ ${ }^{2}$ Departamento de Física Aplicada I, Universidad Complutense, E-28040 Madrid, Spain \\ ${ }^{3}$ GISC, Universidad Complutense, E-28040 Madrid, Spain \\ (Received 12 September 2005; published 9 December 2005)
}

\begin{abstract}
We present a generalization of the Green-Kubo expressions for thermal transport coefficients $\mu$ in complex fluids of the generic form $\mu=\mu_{\infty}+\int_{0}^{\infty} d t V^{-1}\left\langle J_{\epsilon} \exp (t \mathcal{L}) J\right\rangle_{0}$, i.e., a sum of an instantaneous transport coefficient $\mu_{\infty}$, and a time integral over a time correlation function in a state of thermal equilibrium between a current $J$ and its conjugate current $J_{\epsilon}$. The streaming operator $\exp (t \mathcal{L})$ generates the trajectory of a dynamical variable $J(t)=\exp (t \mathcal{L}) J$ when used inside the thermal average $\langle\cdots\rangle_{0}$. These formulas are valid for conservative, impulsive (hard spheres), stochastic, and dissipative forces (Langevin fluids), provided the system approaches a thermal equilibrium state. In general $\mu_{\infty} \neq 0$ and $J_{\epsilon} \neq J$, except for the case of conservative forces, where the equality signs apply. The most important application in the present paper is the hard sphere fluid.
\end{abstract}

DOI: 10.1103/PhysRevE.72.061102

PACS number(s): 05.20.Dd, 05.40.-a

\section{INTRODUCTION}

As the interest in fluids during the last decades has been shifting from standard fluids with smooth conservative interactions to more complex fluids with ditto interactions, a new analysis of linear response theory for such systems seems timely. The present paper provides this analysis for a wide class of complex fluid-type systems.

The Green-Kubo formulas for thermal transport coefficients in simple classical fluids with conservative interactions are widely used, and generally accepted [1-4] as exact expressions for general densities, as long as the deviations from equilibrium and the gradients are small, and the transport coefficients exist. These expressions are given in terms of equilibrium time correlation functions between $\mathrm{N}$-particle currents, i.e.,

$$
\mu=\int_{0}^{\infty} d t \lim _{V \rightarrow \infty} \frac{1}{V}\langle J(0) J(t)\rangle_{0}
$$

where $\mu$ denotes a typical transport coefficient, $\langle\ldots\rangle_{0}$ is an average over a thermal equilibrium ensemble at temperature $T=1 / k_{B} \beta$, with a weight $\rho_{0} \sim \exp [-\beta H]$, and $\lim _{V \rightarrow \infty}$ denotes the thermodynamic limit. In the sequel this limit is understood, but not explicitly written. The time evolution of a dynamical variable $J(t)=e^{t \mathcal{L}} J(0)$ can be described by a streaming operator $e^{t \mathcal{L}}$, and is invariant under the time reversal transformation. The infinitesimal generator $\mathcal{L}$, referred to as Liouville operator, changes sign under the time reversal transformation. The total microscopic flux $J=\lim _{\mathbf{k} \rightarrow 0} j_{\mathbf{k}}$ is related to the Fourier mode $a_{\mathbf{k}}$ of a conserved density through the local conservation law $\partial_{t} a_{\mathbf{k}} \equiv \mathcal{L} a_{\mathbf{k}}=-i \mathbf{k} \cdot \mathbf{j}_{\mathbf{k}}$, and contains in general contributions from kinetic transport, and from collisional transfer, i.e., transport through inter-particle forces. The simple form (1.1) only applies to systems with time reversal invariant dynamics, as we shall see in this paper.

A summary of the results for systems with nonconservative forces has been given in Ref. [5], and the goal of this article is to provide a general analysis with detailed applications to hard sphere fluids. The applications to stochastic systems, as described by Langevin and FokkerPlanck equations will be given elsewhere [6]. Green-Kubo formulas still exist, but their generic form is different from Eq. (1.1). The systems of interest are elastic hard sphere fluids with impulsive forces [7], and systems with Brownian dynamics [8], considered as a mixture of hard spheres where the mass ratio is taken to infinity; lattice gas cellular automata [9] and multiparticle collision dynamics (MPCD) models, where the discrete time dynamics involves stochastic variables [10]. Finally there is the large class of complex fluids, where $N$-particle fluid or magnetic systems are described by mesoscopic Langevin equations containing dissipative and stochastic forces, or by corresponding FokkerPlanck equations for the probability distribution [11-23]. In fact, also the models for critical and unstable fluids and magnets [24] belong to the Langevin models covered by the present theory. We also note that the Langevin and FokkerPlanck equations are not time reversal invariant. The theory to be developed here may be applied as soon as the analytic form of the generator $\mathcal{L}$ and of the thermal distribution $\rho_{0}$ are given, i.e., as soon as the time evolution can be expressed either in terms of hard sphere dynamics or in terms of Langevin and Fokker-Planck equations.

The systems listed above not only refer to simple fluids, but cover a large range of complex fluids, also outside the collection of critical and unstable systems. For instance, Ref. [23] considers diffusion coefficients of colloidal particles, whereas Ref. [22] contains an extensive list of applications of the so-called dissipative particle dynamics (DPD) fluid to colloidal particles, emulsions, and polymers $[11,12]$. These are just a few examples, but there are many more nonequilibrium fluid-type models, that take fluctuations into account, satisfy detailed balance criteria, and reach thermal equilibrium, for which Green-Kubo formulas have been or can be derived.

Granular fluids are outside the scope of this study, although Green-Kubo formulas have been considered in the 
literature $[25,26]$. The reason is that such systems do not approach a stable state of thermal equilibrium, which is a basic requirement in the present derivation. In the above articles the stable equilibrium state is replaced by the unstable homogeneous cooling state. Such extensions are outside the scope of the present paper.

The goal of this study is to derive generalized GreenKubo expressions for large classes of $N$-particle systems, that do approach thermal equilibrium, and have equations of motion that possibly lack time reversal invariance. The interaction forces may be impulsive, dissipative, stochastic, and possibly include conservative forces as well. The method used is linear response theory [1], which yield exact expressions for transport coefficients, valid for arbitrary densities. They have the generic form

$$
\mu=\mu_{\infty}+V^{-1} \int_{0}^{\infty} d t\left\langle J_{\epsilon} e^{t \mathcal{L}} J\right\rangle_{0} .
$$

The pseudostreaming operators $\exp (t \mathcal{L})$ with $t>0$ are well defined for all points in the relevant phase space, and generate the proper trajectories of the dynamical variables when used inside statistical averages $\langle\cdots\rangle_{0}$. An intuitive generalization of Eq. (1.1) to nonconservative forces does not seems obvious. For the general class of Langevin systems a result similar to (1.2) has been obtained in Ref. [23], but the instantaneous contribution $\mu_{\infty}$ to the dissipative transport coefficient has not been identified. The only known results of the complete form (1.2) with instantaneous part as well as time correlation part have been obtained in Ref. [8] for the friction coefficient of Brownian particles, and in Ref. [7] for the shear viscosity of the hard sphere fluid. In the former case the second term in Eq. (1.2) contains a time correlation function of an interparticle force and its conjugate force, in the latter of a stress and a conjugate stress.

A discussion about exact expressions for transport coefficients is not complete without the Einstein-Helfand formulas for the Navier-Stokes transport coefficients [1-3]. They are the analogs of Einstein's formula for the self diffusion $D_{s}$, and are also expressed in equilibrium time correlation functions, e.g.,

$$
\begin{gathered}
D_{s}=\lim _{t \rightarrow \infty}(1 / 2 t)\left\langle[x(t)-x(0)]^{2}\right\rangle_{0} \\
\eta=\lim _{t \rightarrow \infty}(\beta / 2 V t)\left\langle[M(t)-M(0)]^{2}\right\rangle_{0},
\end{gathered}
$$

where $\eta$ is the shear viscosity with $M(t)=\sum_{i} m v_{i y}(t) x_{i}(t)$. In fact these forms give a more compact and more robust representation of transport coefficient than the Green-Kubo formulas (1.1) or (1.2). This is the case because the former can be expressed by only specifying the momentum and energy of a particle, and do not require the explicit introduction of forces. Consequently, the Helfand formulas apply to systems with conservative, impulsive, dissipative and stochastic forces, and either type of Green-Kubo formulas (1.1) or (1.2) can be derived for the relevant cases. An added advantage of the Einstein-Helfand formulas is that they are evidently positive.
So there exist two independent routes for generalizing Green-Kubo formulas to nonconservative forces. The first one is to start from the Einstein-Helfand formulas, and transform them into Eq. (1.2). This route has been followed in Ref. [7]. The second one is to derive the generalizations from the fundamental Liouville equation, or its mesoscopic ana$\log$, the Fokker-Planck equation. The latter route is followed in the present paper.

The plan of the paper is as follows. In Sec. II we obtain a fundamental commutation relation between the $\mathrm{N}$-particle equilibrium distribution function $\rho_{0}$ and the infinitesimal generator $\mathcal{L}$ (Liouville or Fokker-Planck operator), that is required in the derivation of the Green-Kubo formulas. In Sec. III the generic form (1.2) of these formulas is derived using the Zwanzig-Mori projection operator method in a form that is applicable to systems with conservative forces (classical fluids, lattice gas cellular automata, MPCD models), impulsive forces (hard spheres), and stochastic and dissipative forces (Langevin fluids). In Sec. IV we apply the new results to hard sphere fluids with some technical details deferred to the Appendix. Further comments and conclusions are presented in Sec. V.

\section{FUNDAMENTAL COMMUTATION RELATION}

We will analyze the similarities and differences among the systems, mentioned in the introduction, all of which evolve to a thermal equilibrium state described by the $N$-particle equilibrium distribution function $\rho_{0}$. This enables us to formulate a fundamental commutation relation for the infinitesimal generator $\mathcal{L}$, that is required in the derivation of the Green-Kubo formulas for systems whose equations of motion are not time reversal invariant, or more precisely, whose pseudostreaming operators (Langevin fluids, elastic hard spheres) do not generate time reversal invariant dynamics.

Suppose we have constructed for the system of interest the infinitesimal generator $\mathcal{L}$ and the streaming operator $\exp (t \mathcal{L})$, that generates the time evolution of dynamic variables inside averages. To study equilibrium time correlation functions, we need two different types of transposes of $\mathcal{L}$. The first transpose, $\widetilde{\mathcal{L}}$, referred to as adjoint, is defined with respect to an inner product with a weight equal to unity, and a second transpose $\mathcal{L}^{\epsilon}$, referred to as conjugate, is defined with a weight $\rho_{0}$, i.e.,

$$
\begin{gathered}
\int d \Gamma A \mathcal{L} B=\int d \Gamma B \tilde{\mathcal{L}} A, \\
\langle A \mathcal{L} B\rangle_{0}=\int d \Gamma \rho_{0} A \mathcal{L} B \equiv \int d \Gamma \rho_{0} B \mathcal{L}^{\epsilon} A=\left\langle B \mathcal{L}^{\epsilon} A\right\rangle_{0} .
\end{gathered}
$$

The corresponding streaming operators $A(t)=\exp (t \mathcal{L}) A(0)$ and $A(-t)=\exp \left(t \mathcal{L}^{\epsilon}\right) A(0)$ with $t>0$, generate the forward and backward or time reversed evolution inside averages in the sense that equilibrium time correlation functions are stationary, i.e., depend only on time differences 


$$
\langle A(0) B(t)\rangle_{0}=\left\langle A e^{t \mathcal{L}} B\right\rangle=\left\langle B e^{t \tilde{\mathcal{L}}} A\right\rangle_{0}=\langle A(-t) B(0)\rangle_{0} .
$$

Having obtained a conjugate generator $\mathcal{L}^{\epsilon}$, we also introduce a conjugate current $J_{\epsilon}=\lim _{k \rightarrow 0} j_{\epsilon \mathbf{k}}^{*}$ through the relations $\mathcal{L}^{\epsilon} a_{\mathbf{k}}^{*}=-i \mathbf{k} \cdot \mathbf{j}_{\epsilon \mathbf{k}}^{*}$. For conservative forces the $\mathcal{L}$ operators are first-order differential operators. So, the adjoint $\widetilde{\mathcal{L}}=-\mathcal{L}$. The conjugate operator $\mathcal{L}^{\epsilon}$ for conservative interactions follows directly from the second line in Eq. (2.1), because $\mathcal{L}$ and $\rho_{0}$ commute, and the conjugate operator equals the adjoint, i.e., for time reversal invariant dynamics

$$
\mathcal{L}^{\epsilon}=-\mathcal{L}=\widetilde{\mathcal{L}} .
$$

Note that here the conjugate current satisfies $J_{\epsilon}=J$.

In systems, such as lattice gas cellular automata and MPCD models, the dynamics involves stochastic variables, but does not contain any dissipative interactions. The microscopic equations of motion inside averages are time reversal invariant. Here the statistical averages include averages over all realizations of the stochastic variables. The time reversal transform of the Liouville operator $\mathcal{L}^{\epsilon}$ satisfies the relation $\mathcal{L}^{\epsilon}=-\mathcal{L}$, as is the case for conservative systems. This transform is obtained by reversing the sign of all variables odd under the time reversal transformation (e.g., velocities). Of course even variables remain unchanged (e.g., positions, energies).

Next, we consider the Langevin fluids. They are described by $N$-particle Langevin equations [27,28], and reach thermal equilibrium. From these equations the corresponding FokkerPlanck equation for the $N$-particle probability distribution $\rho(\Gamma, t)$ is constructed, which is the fundamental starting point for our linear response theory. The latter is written here [29] as $\partial_{t} \rho=\tilde{\mathcal{L}} \rho$. As we are interested in time correlation functions, it is sufficient to define streaming operators $e^{t \mathcal{L}}$ for the time evolution of dynamical variables inside averages. This may be done through the adjoint

$$
\langle A(t)\rangle=\int d \Gamma A e^{t \tilde{\mathcal{L}}} \rho(\Gamma, 0)=\int d \Gamma \rho(\Gamma, 0) e^{t \mathcal{L}} A=\left\langle e^{t \mathcal{L}} A\right\rangle .
$$

In this formulation averages over the realizations of stochastic forces are already accounted for in the Fokker-Planck equation. So, $\mathcal{L}$ is the adjoint of the Fokker-Planck operator $\tilde{\mathcal{L}}$. Because of the presence of dissipative forces, the FokkerPlanck equation is not time-reversal invariant. Consequently, $\mathcal{L}^{\epsilon} \neq-\mathcal{L}$ (no odd parity in the velocities). The Fokker-Planck operator $\tilde{\mathcal{L}}$ and its adjoint $\mathcal{L}$ contain first and second order derivatives, and also here the adjoint can be simply obtained.

These Langevin equations have been constructed such that the detailed balance condition holds by imposing that the Gibbs' distribution is a steady state solution of the FokkerPlanck equation, i.e., $\partial_{t} \rho_{0}=\tilde{\mathcal{L}} \rho_{0}=0$. Then dissipative and random forces are related by the fluctuation-dissipation theorem. This implies that the Fokker-Planck equation has an $H$-theorem or Lyapunov functional, and that $\rho_{0}$ is the unique steady state solution.
Next consider the construction of the conjugate operator $\mathcal{L}^{\epsilon}$, where $\rho_{0}$ and $\mathcal{L}$ in Eq. (2.1) do not commute. So we introduce an operator $\overline{\mathcal{L}}$ through the commutation relation

$$
\rho_{0} \mathcal{L}=\overline{\mathcal{L}} \rho_{0} .
$$

Having obtained $\overline{\mathcal{L}}$, we insert this relation in line 2 of Eq. (2.1), and obtain the conjugate generator as the adjoint of $\overline{\mathcal{L}}$, i.e.,

$$
\mathcal{L}^{\epsilon}=\tilde{\overline{\mathcal{L}}}
$$

For the Fokker-Planck equations, satisfying the detailed balance criteria, the commutation relation (2.5) has been derived in the literature $[27,28]$ with a general prescription for $\overline{\mathcal{L}}$. Only in the case of conservative interactions, where the equations of motion are time reversal invariant, the equalities $\overline{\mathcal{L}}=\mathcal{L}$ and $J_{\epsilon}=J$ hold. For nonconservative interactions one has $\overline{\mathcal{L}} \neq \mathcal{L}$ and $J_{\epsilon} \neq J$.

As we will see in the next section, the replacement of Eq. (2.3) for a time evolution with time reversal invariance, by the commutation relation (2.5) for a time evolution without, has severe effects on the explicit form of the Green-Kubo formulas for the Langevin fluids, as shown in Eq. (1.2). There are two conspicuous differences with respect to the standard Green-Kubo formulas (1.1). The fluxes $J_{\epsilon}$ and $J$ inside the equilibrium time correlation function are necessarily unequal, as $\mathcal{L}_{\epsilon} \neq-\widetilde{\mathcal{L}}$, and an additional instantaneous transport coefficient $\mu_{\infty}$ appears, that depends solely on equilibrium properties.

Finally we discuss fluids of elastic hard spheres with mass $m$ and diameter $\sigma$. In the kinetic theory of hard sphere fluids the standard notation [7,30-32] is $\mathcal{L}=L_{+}$, and $\mathcal{L}^{\epsilon}=-L_{-}$. Here the pseudostreaming operator $\exp [t \mathcal{L}]=\exp \left[t L_{+}\right]$with $t>0$ generates forward trajectories, and $\exp \left[t \mathcal{L}^{\epsilon}\right]=\exp \left[-t L_{-}\right]$with $t>0$ backward or time reversed trajectories. Furthermore, it has been shown [30] that the correct hard sphere dynamics inside an equilibrium average is generated by the combination $\rho_{0} A(t)=\rho_{0} e^{t L_{+}} A(0)$. Here the presence of the factor $\rho_{0}$ guarantees that a zero weight is given to the unphysical overlapping initial configurations, for which the pseudostreaming operators generate trajectories as well. These trajectories are not time reversal invariant.

From the explicit form of the pseudo-Liouville operator $\mathcal{L}$, as briefly reviewed in Appendix, it is clear that $\mathcal{L}$ is not odd in the velocity variables, i.e., $\mathcal{L}^{\epsilon} \neq-\mathcal{L}$, and consequently the trajectory, generated by the streaming operator is not invariant under the time reversal transformation. A typical example illustrating the lack of time reversal invariance of the hard sphere trajectories, generated by the streaming operators, is a trajectory in $\Gamma$ space starting from an unphysical overlapping initial configuration. Here the overlapping pairs separate within a finite time. This forward trajectory is not retraced when all velocities are reversed, because the pairs, when meeting again, experience a standard hard sphere collision, in which the backward trajectory is deflected from the forward one. 
In the original literature [30] it has also been shown that the hard sphere generators obey for $t>0$ the commutation relation

$$
\rho_{0} e^{t \mathcal{L}}=e^{t \overline{\mathcal{L}}} \rho_{0},
$$

which implies that the time correlation functions are stationary, i.e., depend only a the time difference. In Eq. (A5) the derivation of Eq. (2.7) is briefly recalled. In terms of time correlation functions for dynamic variables $A$ and $B$ the stationarity relation (2.2) also applies, i.e.,

$$
\left\langle A e^{t \mathcal{L}} B\right\rangle_{0}=\int d \Gamma A e^{t \overline{\mathcal{L}}} \rho_{0} B=\left\langle B e^{t \mathcal{L}^{\epsilon}} A\right\rangle_{0},
$$

where the transpose of $\overline{\mathcal{L}}$ is $\mathcal{L}^{\epsilon}$ on account of Eq. (2.6). Finally we observe that these relations also include conservative systems as a special case, in which $\overline{\mathcal{L}}=\mathcal{L}$ on account of Eqs. (2.6) and (2.3). Consequently Eq. (2.5) reduces to the commutation relation $\rho_{0} \mathcal{L} \cdots=\mathcal{L} \rho_{0} \cdots$, implying that $\rho_{0}$ is a stationary solution of the Liouville equation $\partial_{t} \rho=\tilde{\mathcal{L}} \rho=-\mathcal{L} \rho$. However, $\mathcal{L} \rho_{0} \neq 0$ for the Fokker-Planck equation, where $\widetilde{\mathcal{L}} \rho_{0}=0$ in the stationary case.

In summary, the streaming operators for Langevin and hard sphere fluids both lack time reversal invariance, and both evolve towards a thermal equilibrium state, and their time correlation functions are stationary. These fundamental properties guarantee both the commutation relation (2.5) or (2.7), as well as an identical structure of the Green-Kubo formulas for such vastly different systems as hard sphere fluids and Langevin fluids.

\section{ZWANZIG-MORI PROJECTION OPERATOR METHOD}

The goal of this section is to derive for the fluid models, described above, the hydrodynamic equations and NavierStokes transport coefficients by means of the Zwanzig-Mori projection operator method $[1,3]$. This will be done by deriving macroscopic equations for the averages of conserved microscopic densities, or their linear combinations, collectively denoted by $a^{i}(\mathbf{r})$,

$$
a^{i}(\mathbf{r})=\left\{n(\mathbf{r}), g_{\alpha}(\mathbf{r}), e(\mathbf{r})\right\},
$$

or by a subset of these densities. Here $n(\mathbf{r})=\Sigma_{i} \delta\left(\mathbf{r}-\mathbf{r}_{i}\right)$ is the number density, $\mathbf{g}(\mathbf{r})=\sum_{i} m \mathbf{v}_{i} \delta\left(\mathbf{r}-\mathbf{r}_{i}\right)$ the momentum density, and $e(\mathbf{r})=\Sigma_{i} e_{i} \delta\left(\mathbf{r}-\mathbf{r}_{i}\right)$ the energy density, where the single particle energy $e_{i}=\frac{1}{2} m v_{i}^{2}+\frac{1}{2} \Sigma_{j(\neq i)} V\left(\mathbf{r}_{i j}\right)$ or the energy of an internal state. In fact this program has been carried out in Ref. [3] for classical fluids with conservative interactions. We mostly follow the notation and definitions used in that article. Here we apply the Zwanzig-Mori method to the generators of all systems, discussed in the previous sections.

The general equation of motion for the Fourier-Laplace transform of $a^{i}(\mathbf{r}, t)$ is

$$
(z-\mathcal{L}) a_{\mathbf{k} z}=a_{\mathbf{k}}
$$

and Fourier modes are defined as

$$
\begin{gathered}
a_{\mathbf{k}}=\int d \mathbf{r} e^{-i \mathbf{k} \cdot \mathbf{r}}\left[a(\mathbf{r})-\langle a(\mathbf{r})\rangle_{0}\right] \\
a_{\mathbf{k} z}=\int_{0}^{\infty} d t e^{-z t} a_{\mathbf{k}}(t),
\end{gathered}
$$

where $a_{\mathbf{k}}^{i}$ is a linear combination of conserved densities, which is for small $k$ orthogonal with respect to the inner product

$$
\left\langle a_{\mathbf{k}}^{i} \mid a_{\mathbf{k}}^{j}\right\rangle \equiv V^{-1}\left\langle a_{\mathbf{k}}^{i^{*}} a_{\mathbf{k}}^{j}\right\rangle_{0}=\delta_{i j}+O\left(k^{2}\right),
$$

and the asterisk denotes complex conjugation. This enables us to define a projection operator $\mathcal{P}=1-\mathcal{P}_{\perp}$, as

$$
\mathcal{P} b_{\mathbf{k}}=\sum_{\mathbf{q}, j} a_{\mathbf{q}}^{j}\left\langle a_{\mathbf{q}}^{j} \mid b_{\mathbf{k}}\right\rangle=\sum_{j} a_{\mathbf{k}}^{j}\left\langle a_{\mathbf{k}}^{j} \mid b_{\mathbf{k}}\right\rangle .
$$

Here $\left\langle a_{\mathbf{q}}^{j} \mid b_{\mathbf{k}}\right\rangle=\delta_{\mathbf{q}, \mathbf{k}}\left\langle a_{\mathbf{k}}^{j} \mid b_{\mathbf{k}}\right\rangle$ because of translational invariance. Applications of the Zwanzig-Mori method to Eq. (3.2) yields for the projected part $\mathcal{P} a_{\mathbf{k} z}$,

$$
\left[z-\mathcal{P} \mathcal{L} \mathcal{P}-\mathcal{P} \mathcal{L} \mathcal{P}_{\perp}\left(z-\mathcal{P}_{\perp} \mathcal{L} \mathcal{P}_{\perp}\right)^{-1} \mathcal{P}_{\perp} \mathcal{L P}\right] \mathcal{P} a_{\mathbf{k} z}^{m}=a_{\mathbf{k}}^{m},
$$

or in terms of hydrodynamic propagators, $G_{i j}(\mathbf{k}, z)$ $=\left\langle a_{\mathbf{k}}^{i} \mid a_{\mathbf{k} z}^{j}\right\rangle$, we obtain

$$
\sum_{j}\left[z \delta_{i j}+i k \Omega_{i j}(\mathbf{k})+k^{2} U_{i j}(\mathbf{k}, z)\right] G_{j m}(\mathbf{k}, z)=\delta_{i m} .
$$

The hydrodynamic matrix $H_{i j}=i k \Omega_{i j}+k^{2} U_{i j}$, consists of an equilibrium average $\Omega_{i j}$, which is the Euler matrix, and a time correlation function $U_{i j}$, which is the Navier-Stokes transport matrix. Its real part $\operatorname{Re} H_{i j}(\mathbf{k}, z)$, is of $O\left(k^{2}\right)$, and represents the dissipative part, and contains the NavierStokes transport coefficients of shear viscosity $\eta$, bulk viscosity $\zeta$ and heat conductivity $\lambda$. For conservative forces $\operatorname{Im} H_{i j}=k \Omega_{i j}$ and $\operatorname{Re} H_{i j}=k^{2} U_{i j}$. For non-conservative forces $\Omega_{i j}$ contains real and imaginary parts, as we shall see. By diagonalizing the hydrodynamic matrix for small $k$ to $O\left(k^{2}\right)$ one finds the hydrodynamic eigenvalues in the form $z_{\lambda} \simeq$ $-i k c_{\lambda}-k^{2} D_{\lambda}$ and the hydrodynamic modes $a_{\mathbf{k}}^{i}$ as special linear combinations of conserved densities. They will be made explicit below. Here the imaginary part of $z_{\lambda}$ contains the propagation speed $c_{\lambda}$ of that mode (which may be vanishing), and the real part contains the diffusivity or dampings coefficient $D_{\lambda}$ of the mode. We cite the results of Eqs. (44) and (45), obtained in Ref. [3],

$$
\begin{gathered}
D_{\eta}=\eta / \rho=\mathcal{L} \operatorname{im} \operatorname{Re}\left\{i k \Omega_{\eta \eta}(\mathbf{k})+k^{2} U_{\eta \eta}(\mathbf{k}, z)\right\} / k^{2} \\
=-\mathcal{L} \operatorname{im} \operatorname{Re}\left\{\left\langle a_{\mathbf{k}}^{\eta_{i}} \mid \mathcal{L} a_{\mathbf{k}}^{\left.\eta_{i}\right\rangle}\right\rangle+\left\langle\mathcal{L}^{\epsilon} a_{\mathbf{k}}^{\eta_{i} \mid} \mathcal{P}_{\perp} \hat{\mathcal{G}}_{z} \mathcal{P}_{\perp} \mathcal{L} a_{\mathbf{k}}^{\eta_{i}}\right\rangle\right\} / k^{2}, \\
D_{l}=-\mathcal{L} \operatorname{im} \operatorname{Re}\left\{\left\langle a_{\mathbf{k}}^{l} \mid \mathcal{L} a_{\mathbf{k}}^{l}\right\rangle+\left\langle\mathcal{L}^{\epsilon} a_{\mathbf{k}}^{l} \mid \mathcal{P}_{\perp} \hat{\mathcal{G}}_{z} \mathcal{P}_{\perp} \mathcal{L} a_{\mathbf{k}}^{l}\right\rangle\right\} / k^{2} \\
D_{T}=\lambda /\left(n \mathcal{C}_{p}\right)=-\mathcal{L} \operatorname{im} \operatorname{Re}\left\{\left\langle a_{\mathbf{k}}^{T} \mid \mathcal{L} a_{\mathbf{k}}^{T}\right\rangle\right. \\
\left.+\left\langle\mathcal{L}^{\epsilon} a_{\mathbf{k}}^{T} \mid \mathcal{P}_{\perp} \hat{\mathcal{G}}_{z} \mathcal{P}_{\perp} \mathcal{L} a_{\mathbf{k}}^{T}\right\rangle\right\} / k^{2} .
\end{gathered}
$$

Here the symbols $D_{\eta}, D_{l}$, and $D_{T}$ represent, respectively, the 
transverse, longitudinal, and thermal diffusivities, $\rho=m n$ is the mass density, and $\mathcal{C}_{p}$ is the specific heat per particle at constant pressure. Furthermore, $\mathcal{L}$ im represents the double limit, $\lim _{z \rightarrow 0} \lim _{k \rightarrow 0}$. In the equation above $\hat{\mathcal{G}}_{z}$ is the projected resolvent operator

$$
\hat{\mathcal{G}}_{z}=\mathcal{P}_{\perp}\left(z-\mathcal{P}_{\perp} \mathcal{L} \mathcal{P}_{\perp}\right)^{-1} \mathcal{P}_{\perp}
$$

which reduces in the small- $k$ limit to the standard resolvent $\mathcal{P}_{\perp}(z-\mathcal{L})^{-1} \mathcal{P}_{\perp}$ when acting on projected dynamic variables [3]. The transformation of the matrix element $U_{i j}(\mathbf{k}, z)$ from the representation $\left\langle a_{\mathbf{k}}^{i} \mid \mathcal{L} \mathcal{P}_{\perp} \cdots\right\rangle$ in Eq. (3.6) to the representation $\left\langle\mathcal{L}^{\epsilon} a_{\mathbf{k}}^{i} \mid \mathcal{P}_{\perp} \cdots\right\rangle$ in Eq. (3.8) follows directly from the commutation relation (2.5), valid for Langevin- and hard sphere fluids. We also note that the relation applies to systems with dynamics that is time reversal invariant, i.e., where $\mathcal{L}^{\epsilon}=-\mathcal{L}$.

In summary, the equations as listed in Eq. (3.8) are valid for all models discussed in this paper. Through this formulation we have extended the linear response theory for conservative systems in Ref. [3] to more complex fluids such as Langevin and hard sphere fluids.

In order to apply these formulas to different systems the definitions and some properties of the quantities appearing in Eq. (3.8) will be required. Due to the isotropy of simple fluids in thermal equilibrium the $\left(\eta_{i}, \eta_{j}\right)$-matrix elements between shear modes $\left\{a_{\mathbf{k}}^{\eta_{i}}\right\}$ are diagonal and independent of the transverse directions $(i=1,2, \ldots, d-1)$. We further recall that the viscosity in isotropic simple fluids is a fourth rank isotropic tensor, determined by two independent scalars, i.e.,

$$
\eta_{\alpha \beta \gamma \delta}=\eta\left\{\delta_{\alpha \gamma} \delta_{\beta \delta}+\delta_{\alpha \delta} \delta_{\beta \gamma}-(2 / d) \delta_{\alpha \beta} \delta_{\gamma \delta}\right\}+\zeta \delta_{\alpha \beta} \delta_{\gamma \delta} .
$$

They are the shear and bulk viscosity $\eta$ and $\zeta$, respectively, or alternatively the transverse and longitudinal diffusivity $D_{\eta}$ and $D_{l}$ determined by

$$
\begin{gathered}
\eta_{x y x y}=\rho D_{\eta}=\eta, \\
\eta_{x x x x}=\rho D_{l}=2 \eta\left(1-d^{-1}\right)+\zeta .
\end{gathered}
$$

For fluids with conserved densities $n(\mathbf{r}), g_{\alpha}(\mathbf{r})$, and $e(\mathbf{r})$-i.e., classical fluids with conservative interactions, and hard sphere fluids with impulsive interactions - the explicit expressions for the hydrodynamic modes are $[3,33]$

$$
\begin{gathered}
a_{\mathbf{k}}^{\eta_{i}}=\sqrt{\frac{\beta}{\rho}} \mathbf{g}_{\mathbf{k}} \cdot \hat{\mathbf{k}}_{\perp}^{i}(i=1,2, \ldots, d-1), \\
a_{\mathbf{k}}^{\sigma}=\sqrt{\frac{\beta}{2 \rho}}\left\{\frac{1}{c} p_{\mathbf{k}}+\sigma g_{\mathbf{k}}^{l}\right\} \quad(\sigma= \pm 1), \\
a_{\mathbf{k}}^{T}=\sqrt{\frac{\beta}{n T C_{p}}}\left\{e_{\mathbf{k}}-h n_{\mathbf{k}}\right\},
\end{gathered}
$$

where $c$ is the adiabatic speed of sound with $c^{2}=(\partial p / \partial \rho)_{s}$ and $p_{\mathbf{k}}$ is the thermodynamic pressure fluctuation

$$
p_{\mathbf{k}}=\left(\frac{\partial p}{\partial n}\right)_{e} n_{\mathbf{k}}+\left(\frac{\partial p}{\partial e}\right)_{n} e_{\mathbf{k}} .
$$

Furthermore $\left\{\hat{\mathbf{k}}, \hat{\mathbf{k}}_{\perp}^{i}\right\}(i=1,2, \ldots, d-1)$ is a set of $d$ orthonormal unit vectors, where the longitudinal direction $\hat{\mathbf{k}}$ is taken parallel to the $x$ axis for convenience. Moreover, $g_{\mathbf{k}}^{l}=\hat{\mathbf{k}} \cdot \mathbf{g}_{\mathbf{k}}$ is the longitudinal component of $\mathbf{g}_{\mathbf{k}}$. Here $a_{\mathbf{k}}^{\eta_{i}}$ are the shear or transverse velocity modes, $a_{\mathbf{k}}^{\sigma}(\sigma= \pm)$ the sound modes, and $a_{\mathbf{k}}^{T}$ the heat mode.

It remains to work out the expressions (3.8) for the different fluid models, using the appropriate hydrodynamic modes $a_{\mathbf{k}}^{i}$ in Eq. (3.12), in combination with the explicit forms in the pseudo-Liouville operator for these models.

Equations (3.8) are the main new results of the general part of this paper. We note the latent presence of the structure of Eq. (1.2), where the instantaneous transport coefficient $\mu_{\infty} \propto \operatorname{Re}\left\langle a_{\mathbf{k}} \mid \mathcal{L} a_{\mathbf{k}}\right\rangle$, and the time correlation functions with the different currents $J_{\epsilon}$ and $J$ are contained in the matrix $U(\mathbf{k}, z)$. Inserting the currents, defined for small $k$ through

$$
\begin{gathered}
\mathcal{L} a_{\mathbf{k}}=-i k J+O\left(k^{2}\right) \\
\mathcal{L}^{\epsilon} a_{\mathbf{k}}^{*}=-i k J_{\epsilon}+O\left(k^{2}\right),
\end{gathered}
$$

into Eq. (3.8) reduces this equation to the generic structure (1.2), and we recall that the inner product in Eq. (3.8) involves complex conjugation. Consider first the case of conservative forces. There the dynamics is time reversal invariant, $\mathcal{L}^{\epsilon}=-\mathcal{L}$, i.e., $\mathcal{L}$ is odd in the velocity variables, and the contributions of the real part of the $\Omega$-matrix necessarily vanishes, i.e., $\mu_{\infty}=0$. Moreover, both currents, as defined in Eq. (3.14), are equal, $J_{\epsilon}=J$.

In case the forces are not conservative, i.e., $\mathcal{L}^{\epsilon} \neq-\mathcal{L}$, then $\mu_{\infty} \propto \operatorname{Re} k^{-2}\left\langle a_{\mathbf{k}} \mid \mathcal{L} a_{\mathbf{k}}\right\rangle$ is necessarily nonvanishing, and approaches a finite value in the small $k$ limit. Moreover, it follows from general considerations that $J_{\epsilon}$ and $J$ are unequal, both for impulsive forces (hard sphere fluids), and for dissipative forces (Langevin fluids). The explicit expressions will be discussed in Ref. [6].

In hindsight the instantaneous transport coefficients $\mu_{\infty}$ can be identified independently of the present analysis. In a kinetic theory description, $\mu_{\infty}$ comes from the irreversible part of the average of the microscopic or mesoscopic $N$-particle current $\langle J\rangle_{\text {loc }}$ in a state of local equilibrium, i.e., the part of $\langle J\rangle_{\text {loc }}$ that contributes to the irreversible entropy production. In the context of complex fluids this is the part that contributes to the Navier-Stokes transport matrix in the hydrodynamic equations. For instance, in the momentum current or stress tensor one finds both in the hard sphere fluid $[33,34]$, as well as in Langevin fluids [13], $\left\langle J_{x y}\right\rangle_{\mathrm{loc}}=\rho u_{x} u_{y}$ $-\eta_{\infty} \nabla_{x} u_{y}$. Here the first term $\rho u_{x} u_{y}$ is the Euler or reversible part of the current, and the second term is the irreversible part, yielding a contribution $\eta_{\infty}$ to the Navier-Stokes transport coefficients. For the hard sphere fluid the statement above will be explicitly verified in Sec. IV. For the Langevin fluids and solids it will be verified in Ref. [6]. As the concept of local equilibrium state is intuitively clear, one can, in principle, also estimate $\mu_{\infty}$ for more complex fluids. 
Perhaps the most intuitive understanding of $\mu_{\infty}$ has been given by Hoogerbrugge and Koelman [11] when first introducing the dissipative particle dynamics (DPD) model. The estimate of the shear viscosity of the DPD fluid $\eta \simeq \eta_{\infty}$ by making a continuum (macroscopic) approximation of the Langevin equation of motion. We finally note that the instantaneous transport coefficient is also one of the main contributions to the transport coefficients of hard sphere and Langevin fluids at liquid densities.

The appearance of two different currents $J$ and $J_{\epsilon}$ in the Green-Kubo formulas for the viscosity and friction coefficient in hard sphere fluids have been derived before in Refs. $[7,8]$. For an gas of inelastic hard spheres similar results have been obtained in Ref. [26]. For a general class of FokkerPlanck equations, satisfying detailed balance, local macroscopic evolution equations have been derived in Ref. [23]. In doing so the expressions, derived for the transport coefficients, are also given in the form of Green-Kubo formulas with two different currents $J$ and $J_{\epsilon}$. However, the relation between $J_{\epsilon}$ and $J$ in that case is more complicated than in the cases discussed in the present paper. This ends the general part of the paper. The remainder deals with the most important application of the present theory, the hard sphere fluid.

\section{CLASSICAL AND HARD SPHERE FLUIDS}

\section{A. Classical conservative fluids}

In the application part of this paper we first list for later reference the Green-Kubo formulas in conservative classical fluids, and next we work out the details of the Green-Kubo formulas for hard sphere fluids. For classical fluids with conservative interactions the explicit forms have been derived in Ref. [3]. Here the Liouville operator is odd in the velocities because of time reversal invariance. Consequently the diagonal elements $\Omega_{\eta \eta}$ and $\Omega_{T T}$ of the Euler matrix in Eq. (3.8) are vanishing. Computation of the elements of the NavierStokes matrix yields for the shear viscosity $\eta$, longitudinal viscosity $D_{l}$, and heat conductivity $\lambda[3]$,

$$
\begin{gathered}
\eta=(\beta / V) \int_{0}^{\infty} d t\left\langle\delta S_{x y}(0) \delta S_{x y}(t)\right\rangle_{0} \equiv \int_{0}^{\infty} d t C_{\eta}(t), \\
\rho D_{l}=(\beta / V) \int_{0}^{\infty} d t\left\langle\delta S_{x x}(0) \delta S_{x x}(t)\right\rangle_{0} \equiv \int_{0}^{\infty} d t C_{l}(t), \\
\lambda=(\beta / T V) \int_{0}^{\infty} d t\left\langle Q_{x}(0) Q_{x}(t)\right\rangle_{0} \equiv \int_{0}^{\infty} d t C_{T}(t)
\end{gathered}
$$

with the subtracted microscopic fluxes

$$
\begin{gathered}
\delta S_{x y}=\mathcal{P}_{\perp} J_{x y}=J_{x y}=\sum_{i} m v_{i x} v_{i y}+\sum_{i<j} r_{i j, x} F_{i j, y}, \\
\delta S_{x x}=\mathcal{P}_{\perp}\left(J_{x x}-\left\langle J_{x x}\right\rangle_{0}\right) \\
=J_{x x}-p V-\left(\frac{\partial p}{\partial e}\right)_{n}\left(H-\langle H\rangle_{0}\right)-\left(\frac{\partial p}{\partial n}\right)_{e}\left(N-\langle N\rangle_{0}\right),
\end{gathered}
$$

$$
\begin{gathered}
Q_{x}=\mathcal{P}_{\perp} J_{x}=J_{x}-P_{x}\left\langle P_{x} J_{x}\right\rangle_{0} /\left\langle P_{x} P_{x}\right\rangle_{0}=J_{x}-\frac{h}{m} P_{x}, \\
J_{x}=\sum_{i} e_{i} v_{i x}+\sum_{i<j}\left(\mathbf{v}_{i}+\mathbf{v}_{j}\right) \cdot \mathbf{F}_{i j} r_{i j, x} .
\end{gathered}
$$

Here $J_{x \alpha}$ is the microscopic $N$-particle momentum flux or stress tensor, where $F_{i j, \alpha}=-\partial V\left(r_{i j}\right) / \partial r_{i j, \alpha}$ with $\alpha=x, y, \ldots, d$ is the conservative interparticle force and $V\left(r_{i j}\right)$ the spherically symmetric pair potential, $\left\langle J_{x x}\right\rangle_{0}=p V$ is the hard sphere pressure in equilibrium, $h=(e+p) / n$ is the enthalpy per particle, and $P_{\alpha}=\Sigma_{i} m v_{i \alpha}$ the total momentum. Moreover, $J_{\alpha}$ is the total energy flux. The quantity $P_{\alpha} h / m$, which is the component of $J_{\alpha}$ parallel to the conserved quantity $P_{\alpha}$, is subtracted out in $Q_{x}$ [3].

\section{B. Hard spheres: Instantaneous transport coefficients}

This section deals with the $\Omega$ matrix, $\left\langle a_{\mathbf{k}}^{i} \mid \mathcal{L} a_{\mathbf{k}}^{i}\right\rangle$, in which the first major difference in the Green-Kubo formulas shows up between systems with time reversal invariance, i.e., $\mathcal{L}^{\epsilon}=$ $-\mathcal{L}$, and those without, i.e., $\mathcal{L}^{\epsilon} \neq-\mathcal{L}$. So, in hard sphere systems the Liouville operators necessarily contain even parts in the velocities-apart from possible odd parts. The matrix elements of $\mathcal{L}$ always have real parts of $O\left(k^{2}\right)$, that contribute to the irreversible or Navier-Stokes part of the hydrodynamic equations. These terms are referred to as the instantaneous or high frequency parts of the transport coefficients. The appearance of such terms is a very robust feature of all systems with streaming operator that lack time reversal invariance. We also recall that the streaming operator $\exp (t \mathcal{L})$ in Eq. (3.8) have to be defined for all points in phase space, including the physically inaccessible overlapping ones.

For hard sphere fluids the instantaneous contributions, which are averages in thermal equilibrium, are calculated in Appendix, and yield results, exact for a $d$-dimensional hard sphere fluids at general densities

$$
\begin{gathered}
\eta_{\infty}=\varpi d /(d+2), \\
\lambda_{\infty}=\varpi d k_{B} /(2 m) \equiv \mathcal{C}_{V} \varpi, \\
\rho D_{l \infty}=3 \varpi d /(d+2), \\
\zeta_{\infty}=\varpi .
\end{gathered}
$$

For completeness we have also listed the instantaneous contribution to the longitudinal diffusivity and the bulk viscosity, which have been obtained similarly with the help of Eq. (3.11). In the equations above we have introduced

$$
\begin{gathered}
\varpi=\rho \sigma^{2} / d^{2} t_{E}, \\
t_{E}=\sigma \sqrt{\pi \beta m} / 2 d b n \chi,
\end{gathered}
$$

where the definition of $\varpi$ has been chosen such that it coincides for $d=3$ with the notation used in Ref. [34], and $t_{E}$ is the Enskog mean free time, $\chi=g_{0}\left(r=\sigma^{+}\right)$is the value of the radial distribution at contact, $b=(1 / 2 d) \Omega_{d} \sigma^{d}$ is the excluded 
volume which equals half the volume of an action sphere with radius $\sigma$, and $\Omega_{d}=2 \pi^{d / 2} / \Gamma(d / 2)$ is the surface area of a $d$-dimensional unit sphere. Alternatively these parameters may be expressed in terms of the equation of state for hard spheres through $\beta p=n(1+b n \chi)$.

\section{Hard spheres: Time correlation functions}

The Navier-Stokes matrix $\lim _{\mathbf{k} \rightarrow 0} U(\mathbf{k}, z)=U(z)$ gives in the long wave length limit the frequency-dependent $(z=i \omega)$ transport coefficients (memory effects). In the previous subsection we have been dealing with the contributions $\eta_{\infty}, \zeta_{\infty}, \lambda_{\infty}$ at infinite frequency. We introduce $\delta C_{a}(t)$ to denote the inverse Laplace transform of the Navier-Stokes part of the hydrodynamic matrix $\rho U_{a a}(z) \equiv \delta \widetilde{C}_{a}(z)$ with $a$ $=\{\eta, l, T\}$. Using the explicit forms (3.12) for the hydrodynamic modes we obtain from Eq. (3.8) the Laplace transforms of the time correlation functions

$$
\begin{gathered}
\rho U_{\eta \eta}(z) \equiv \delta \widetilde{C}_{\eta}(z)=-\lim _{k \rightarrow 0} \frac{\beta}{k^{2} V}\left\langle\left(\mathcal{L}^{\epsilon} g_{\mathbf{k} y}^{*}\right) \mathcal{P}_{\perp} \hat{\mathcal{G}}_{z} \mathcal{P}_{\perp} \mathcal{L} g_{\mathbf{k} y}\right\rangle_{0}, \\
\rho U_{l l}(z) \equiv \delta \widetilde{C}_{l}(z)=-\lim _{k \rightarrow 0} \frac{\beta}{k^{2} V}\left\langle\left(\mathcal{L}^{\epsilon} g_{\mathbf{k} x}^{*}\right) \mathcal{P}_{\perp} \hat{\mathcal{G}}_{z} \mathcal{P}_{\perp} \mathcal{L} g_{\mathbf{k} x}\right\rangle_{0}, \\
n \mathcal{C}_{p} U_{T T}(z) \equiv \delta \widetilde{C}_{T}(z)=-\lim _{k \rightarrow 0} \frac{\beta}{k^{2} T V}\left\langle\left(\mathcal{L}^{\epsilon} e_{\mathbf{k}}^{*}\right) \mathcal{P}_{\perp} \hat{\mathcal{G}}_{z} \mathcal{P}_{\perp} \mathcal{L} e_{\mathbf{k}}\right\rangle_{0} .
\end{gathered}
$$

The asterisk denotes complex conjugation. From here on only the standard notation of hard sphere kinetic theory [7,30-32] will be used with $\mathcal{L}=L_{+}$and $\mathcal{L}^{\epsilon}=-L_{-}$. To compute the explicit form of the currents we use the relation

$$
\mathbf{k} b_{\mathbf{k}}=\mathbf{k} \delta b_{\mathbf{k}}=\mathbf{k}\left(b_{\mathbf{k}}-\delta_{\mathbf{k}, 0}\left\langle b_{0}\right\rangle_{0}\right)=\mathbf{k} \delta b_{0}+O\left(k^{2}\right),
$$

where the fluctuation $\delta b_{\mathbf{k}}=b_{\mathbf{k}}-\left\langle b_{\mathbf{k}}\right\rangle_{0}$. Here we have used the relation $\left\langle b_{\mathbf{k}}\right\rangle_{0}=\delta_{\mathbf{k}, 0}\left\langle b_{0}\right\rangle_{0}$, which is a consequence of translational invariance. The replacement of $b_{\mathbf{k}}$ by $\delta b_{\mathbf{k}}$ in Eq. (4.6) is convenient for taking the limit $\mathbf{k} \rightarrow 0$. It is further convenient to choose $\mathbf{k}$ parallel to the $x$ axis. Consider first the longitudinal $(\alpha=x)$ and transversal $(\alpha=y)$ momentum current. Then we obtain with the help of Eq. (4.6) for small $k$,

$$
\begin{gathered}
\mathcal{L} g_{\mathbf{k} \alpha}=L_{+} g_{\mathbf{k} \alpha}=-i k \delta J_{+x \alpha}=-i k\left(\delta J_{x \alpha}^{k}+\delta J_{+x \alpha}^{v}\right), \\
\mathcal{L}^{\epsilon_{\mathbf{k} \alpha}^{*}}=-L_{-} g_{\mathbf{k} \alpha}^{*}=-i k \delta J_{-x \alpha}=-i k\left(\delta J_{x \alpha}^{k}+\delta J_{-x \alpha}^{v}\right) .
\end{gathered}
$$

By inserting the expressions for the hard sphere generators in Eq. (A1), and using the properties (A2)-(A6) it is straightforward to obtain the kinetic part $(k)$ and collisional transfer part $(v)$ of the momentum currents

$$
J_{x \alpha}^{k}=\sum_{i} m v_{i x} v_{i \alpha}
$$

$$
\begin{aligned}
J_{ \pm x \alpha}^{v} & = \pm \sum_{i<j} T_{ \pm}(i j) m\left(v_{i \alpha} r_{i x}+v_{j \alpha} r_{j x}\right) \\
& =\sum_{i<j} m \sigma^{d} \int^{(\mp)} d \hat{\sigma}\left(\mathbf{v}_{i j} \cdot \hat{\sigma}\right)^{2} \hat{\sigma}_{x} \hat{\sigma}_{\alpha} \delta\left(\mathbf{r}_{i j}-\sigma \hat{\sigma}\right) .
\end{aligned}
$$

The averages of the fluxes are only nonvanishing for $\alpha=x$, i.e.,

$$
\begin{gathered}
\left\langle J_{x x}^{k}\right\rangle_{0}=V n / \beta, \quad \text { and }\left\langle J_{ \pm x x}^{v}\right\rangle_{0}=V n^{2} b \chi / \beta, \\
\left\langle J_{ \pm x x}\right\rangle_{0}=V n(1+b n \chi) / \beta=V p
\end{gathered}
$$

in complete agreement with the result (4.2) for smooth potentials. In the transition from the second to the third line in Eq. (4.8) one may use Eqs. (A8) and (A2) and express the variables $\left\{\mathbf{v}_{i}, \mathbf{r}_{i}, \mathbf{v}_{j}, \mathbf{r}_{j}\right\}$ in center-of-mass coordinates $\left\{\mathbf{R}_{i j}, \mathbf{G}_{i j}\right\}$, and relative ones $\left\{\mathbf{r}_{i j}, \mathbf{v}_{i j}\right\}$, and apply Eq. (A6) for conserved variables. Consider first the transverse stress fluctuation with $\alpha=y$. Here we note that $\mathcal{P}_{\perp} J_{ \pm x y}=J_{ \pm x y}$ in Eq. (4.5), as the (xy) component of the microscopic stress tensor has no subtracted parts. The momentum fluxes $J_{+x y}^{v}$ and $J_{-x y}^{v}$, differ only from one another in the constraints on the integral over the $d$-dimensional solid angle $d \hat{\sigma}$, denoted by the superscript $(-)$ or $(+)$. Here $(\mp)$ requires $(\mp) \mathbf{v}_{i j} \cdot \hat{\sigma}>0$, and implies that the $\hat{\sigma}$ integration is restricted to the precollision hemisphere $(-)$, or to the post-collision hemisphere $(+)$.

The time correlation function for the stresses for $t>0$ can now be written as

$$
\delta C_{\eta}(t)=\frac{\beta}{V}\left\langle J_{-x y} e^{t L_{+}} J_{+x y}\right\rangle_{0}=\frac{\beta}{V}\left\langle J_{+x y} e^{-t L_{-}} J_{-x y}\right\rangle_{0}=\sum_{a, b} \delta C_{\eta}^{a b}(t),
$$

where $a, b=\{k, v\}$. The second equality has been obtained by renaming the dummy variables $\mathbf{v}_{i}$ in $-\mathbf{v}_{i}$ and using Eq. (A1). Also note that the cross-correlations are equal, $\delta C_{\eta}^{k v}(t)$ $=\delta C_{\eta}^{v k}(t)$, as follows directly from the commutation relation (2.8) for hard sphere fluids, where $\mathcal{L}=L_{+}$and $\mathcal{L}^{\epsilon}=-L_{-}$, i.e.,

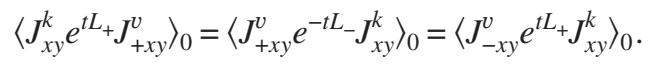

The last equality has again been obtained by renaming the dummy integration variables $\mathbf{v}_{i}$ to $-\mathbf{v}_{i}$. The final Green-Kubo formula for the shear viscosity in a hard sphere fluid follows then from Eq. (3.8), by taking the small- $z$ limit of the frequency dependent $\delta \widetilde{C}_{\eta}(z)$ and combining it with the instantaneous viscosity in Eq. (4.3) to yield the equivalent representations of the generic structure (1.2)

$$
\eta=\eta_{\infty}+(\beta / V) \int_{0}^{\infty} d t\left\langle J_{-x y} e^{t L_{+}} J_{+x y}\right\rangle_{0} .
$$

The hard sphere result for the shear viscosity as well as Eqs. (4.7)-(4.10) have been derived already in Ref. [7], by reinterpreting the Einstein-Helfand formulas in terms of pseudoLiouville propagators.

For the heat conductivity the analysis proceeds along similar lines, and the energy flux is calculated from the Fourier mode of the energy density $e_{k}$, in Eq. (3.1) with $e_{i}$ $=\frac{1}{2} m v_{i}^{2}$, i.e., 


$$
\mathcal{L} e_{\mathbf{k}}=-i k J_{+x}=-i k\left(J_{x}^{k}+J_{+x}^{v}\right),
$$

and a relation for $J_{-x}$ similar to the second line in Eq. (4.7). The subtracted flux is the same as in Eq. (4.2) for conservative fluids, where the enthalpy per particle for hard spheres is $h=h^{k}+h^{v}$ with $e=\frac{1}{2} d n / \beta$ and $p=(n / \beta)(1+b n \chi)$, and where we have used the relation $\left\langle P_{x} J_{ \pm x}\right\rangle=V n^{2} b \chi / \beta^{2}=h^{v} n V / \beta$. The kinetic and collisional parts are then given by

$$
\begin{aligned}
Q_{x}^{k}= & \mathcal{P}_{\perp} J_{x}^{k}=\sum_{i} \frac{1}{2}\left[m v_{i}^{2}-(d+2) / \beta\right] v_{i x}, \\
Q_{ \pm x}^{v}=\mathcal{P}_{\perp} J_{ \pm x}^{v}= & \pm \frac{1}{2} m \sum_{i<j} T_{ \pm}(i j)\left(v_{i}^{2} r_{i x}+v_{j}^{2} r_{j x}\right)-\left(h^{v} / m\right) P_{x} \\
= & \sum_{i<j} m \sigma^{d} \int^{(\mp)} d \hat{\sigma}\left(\mathbf{v}_{i j} \cdot \hat{\sigma}\right)^{2}\left(\mathbf{G}_{i j} \cdot \hat{\sigma}\right) \\
& \times \hat{\sigma}_{x} \delta\left(\mathbf{r}_{i j}-\sigma \hat{\sigma}\right)-(b n \chi / \beta m) P_{x},
\end{aligned}
$$

where $\mathbf{G}_{i j}=\frac{1}{2}\left(\mathbf{v}_{i}+\mathbf{v}_{j}\right)$. The time correlation function of the subtracted heat flux is then

$$
\delta C_{T}(t)=\frac{\beta}{T V}\left\langle Q_{-x} e^{t L_{+}} Q_{+x}\right\rangle_{0}=\sum_{a b} \delta C_{T}^{a b}(t),
$$

and the cross-correlations $(k v)$ and $(v k)$ are again equal [see Eq. (4.11)]. The total heat conductivity in a hard sphere fluid is then similarly given by the Green-Kubo formula

$$
\lambda=\lambda_{\infty}+(\beta / T V) \int_{0}^{\infty} d t\left\langle Q_{-x} e^{t L_{+}} Q_{+x}\right\rangle_{0},
$$

which has again the generic structure (1.2). Obviously, this formula can also be derived from the Einstein-Helfand formulas, following the method of Ref. [7].

For the longitudinal diffusivity $\rho D_{l}$ and the total bulk viscosity $\zeta$ we obtain similarly with the help of Eqs. (4.6)-(4.9)

$$
\begin{gathered}
\rho D_{l}=\rho D_{l \infty}+(\beta / V) \int_{0}^{\infty} d t\left\langle\delta S_{-x x} e^{t L_{+}} \delta S_{+x x}\right\rangle_{0}, \\
\zeta=\zeta_{\infty}+(\beta / V) \int_{0}^{\infty} d t\left\langle\delta S_{\_} e^{t L_{+}} \delta S_{+}\right\rangle_{0},
\end{gathered}
$$

where the subtracted fluxes are

$$
\begin{gathered}
\delta S_{ \pm}=\frac{1}{d} \sum_{\alpha} \delta S_{ \pm \alpha \alpha}, \\
\delta S_{ \pm x x}=\mathcal{P}_{\perp} \delta J_{ \pm x x}=\delta J_{+x x}-\left(\frac{\partial p}{\partial e}\right)_{n} \delta H-\left(\frac{\partial p}{\partial n}\right)_{e} \delta N
\end{gathered}
$$

in complete agreement with the subtracted fluxes in Eq. (4.2). Before concluding this section we note that the instantaneous or high frequency transport coefficients are also present in the Enskog theory for dense hard sphere fluids, as the local equilibrium averages of the collisional transfer parts in Eqs. (4.8) and (4.14) for the corresponding currents, i.e.,

$$
\begin{gathered}
\left\langle J_{+x y}^{v}\right\rangle_{\mathrm{loc}}=-\eta_{\infty} \nabla_{x} u_{y}, \\
\left\langle J_{+x}^{v}\right\rangle_{\mathrm{loc}}=-\lambda_{\infty} \nabla_{x} T, \\
\sum_{\alpha}\left\langle J_{+\alpha \alpha}^{v}\right\rangle_{\mathrm{loc}}=p-\zeta_{\infty} \nabla \cdot \mathbf{u},
\end{gathered}
$$

where the bulk viscosity appears as a correction to the local equilibrium hard sphere pressure $p$, and $\mathbf{u}$ is the local flow field. As the collisional transfer terms are sums over pairs of particles, the averages contain the pair distribution function in local equilibrium, and involves integrations over $\left\{\mathbf{v}_{1}, \mathbf{v}_{2}, \mathbf{r}_{12}\right\}$. They have been calculated in Refs. [33-35] for the special case of $d=3$ as $\eta=(3 / 5) \varpi, \lambda=\mathcal{C}_{V} \varpi$ and $\zeta=\varpi$, and can for instance be found in Eqs. $(16.14,8),(16.42,3)$, and (16.41.7) of Ref. [34] in complete agreement with the results derived here. The explicit expression for $\varpi$ in Eq. (4.4) is proportional to $n^{2}$. Consequently at liquid densities the instantaneous transport coefficients are major contributions, and account for about half the value of the transport coefficient.

For comparison we quote the complete prediction of the Enskog theory for the viscosities of a $d$-dimensional hard sphere fluid. For the bulk viscosity one has $\zeta=\zeta_{\infty}=\varpi$, and for the shear viscosity

$$
\eta=\frac{1}{\chi}\left(1+\frac{2}{d+2} b n \chi\right)^{2} \eta_{0}+\frac{d}{d+2} \varpi .
$$

Here $\eta_{0}$ is the low density Boltzmann value of the shear viscosity [34]. The expression above shows that the Enskog theory gives for the shear viscosity other contributions of order $n^{2}$ at high densities, and that the Enskog prediction for the instantaneous viscosity $\eta_{\infty}=d \varpi /(d+2)$ is exact for all densities.

\section{CONCLUSIONS}

The main result of this paper is a generalization of the standard Green-Kubo or time correlation expressions in Eq. (3.8) for classical fluids with conservative interactions to hard sphere fluids with impulsive interactions, and to mesoscopic Langevin fluids with dissipative and stochastic interactions.

In the classes of fluids above the time dependence of dynamical variables $A(t)=\exp [t \mathcal{L}] A(0)$, can be represented by streaming operators or generators $\exp [t \mathcal{L}]$ when used inside ensemble averages. In conservative systems the dynamics generated by the (pseudo)streaming operators is time reversal invariant, implying $\mathcal{L}^{\epsilon}=-\mathcal{L}$ [see Eq. (2.3)]. This property is necessary to obtain Green-Kubo formulas of the standard form (1.1). In the remaining classes the dynamics generated by the (pseudo)streaming operator is not time reversal invariant, i.e., the backward streaming operator $\exp \left(t \mathcal{L}^{\epsilon}\right)$ $\neq \exp (-t \mathcal{L})$ (with $t>0$ ).

The common criteria, obeyed by the models above are that the systems approach a thermal equilibrium state $\rho_{0}$ 
$\sim \exp [-\beta H]$ and that the corresponding correlation functions $\left\langle A(t) B\left(t^{\prime}\right)\right\rangle_{0}$ are stationary, i.e., only depend on time differences. The criteria imply that the infinitesimal generators for hard sphere and Langevin fluids obey the commutation relation (2.5), i.e., $\rho_{0} \mathcal{L}=\overline{\mathcal{L}} \rho_{0}$. This includes in fact conservative systems as the special case with $\overline{\mathcal{L}}=\mathcal{L}$.

There are two conspicuous differences between GreenKubo formulas in these three classes of fluids. In the classes without time reversal invariant dynamics, i.e., $\mathcal{L}^{\epsilon} \neq-\mathcal{L}$, there is necessarily an instantaneous nonvanishing contribution $\mu_{\infty}$, and in the classes with, i.e., $\mathcal{L}^{\epsilon}=-\mathcal{L}$, the generator is odd in the velocity variables, and $\mu_{\infty}$ automatically vanishes [compare Eqs. (4.12) and (4.16) with Eq. (4.1)]. The next difference concerns the microscopic fluxes. In the classes with time reversal invariance the Green-Kubo formula in Eq. (1.1) is a time integral over an autocorrelation function $\langle J(0) J(t)\rangle_{0}$, containing the same currents. In the cases without, the two currents in $\left\langle J_{\epsilon} e^{t \mathcal{L}} J\right\rangle_{0}$ are unequal. Both types of differences apply to the hard sphere fluids, as well as the Langevin fluids. In the former case our results confirm and extend the results in Ref. [7].

A summary of the Green-Kubo formulas for transport coefficients in Langevin fluids can be found in Ref. [5], and a detailed analysis will be given in Ref. [6], where the results (3.8) will be applied to a Langevin-type solid showing heat conduction, and a similar fluid showing viscosity.

Finally, to understand the origin of the instantaneous contributions in the case of a hard sphere fluid it is instructive to write the shear viscosity (4.12) in the standard form (4.1) for conservative interactions, where the time correlation function for $t>0$ is defined as

$$
C_{\eta}(t)=2 \eta_{\infty} \delta(t)+(\beta / V)\left\langle J_{-x y} e^{t L_{+}} J_{+x y}\right\rangle_{0},
$$

and $\delta(t)$ is a Dirac delta function. It is then of interest to compare this expression with the stress-stress correlation function $C_{\eta}(t)$ in Eq. (4.1), as recently calculated in Ref. [7] for a conservative fluid with a repulsive power law potential $V(r) \sim r^{-\nu}$ (soft spheres) at short times at large values of $\nu$. The short time dynamics has been calculated exactly for the (very short) mean traversal time $\tau_{\nu} \sim 1 / \nu$, required for particles to traverse the steep part of the repulsive potential. It was shown that for asymptotically large $\nu$, the short time behavior of $C_{\eta}(t)$ crosses over to a contribution $\sim \delta(t)$, and agrees with the representation (5.1). This also demonstrates that smooth conservative potentials $r^{-\nu}$ in the singular hard sphere limit as $\nu \rightarrow \infty$, generate a $\delta(t)$-type short time correlation, in agreement with the present results for hard spheres, which have been derived from the very starting point for impulsive interactions.

\section{ACKNOWLEDGMENTS}

M.H.E. is supported by Secretaría de Estado de Educación y Universidades (Spain), and R.B. by the Universidad Complutense (Profesores en el Extranjero). This work is financed by the research project FIS2004-271 (Spain).

\section{APPENDIX: HARD SPHERE DYNAMICS}

\section{Pseudo-Liouville and binary collision operators}

The time evolution of any phase space function $A(t)$ $=e^{t \mathcal{L}} A(0)$, evolving under the hard sphere dynamics [30], can be described for positive times $(t>0)$ by the forward pseudostreaming operator $\exp \left[t L_{+}\right]$, where $\mathcal{L}=L_{+}$is the forward pseudo-Liouville operator for hard spheres. For backward or time-reversed dynamics the pseudostreaming operator is $e^{t \mathcal{L}^{\epsilon}}=e^{-t L_{-}}(t>0)$. While true hard sphere dynamics is undefined for physically inaccessible overlapping configurations (with any $r_{i j}<\sigma$ ), the pseudo-streaming operators are defined for all overlapping and nonoverlapping configurations. In the latter they generate the true hard sphere dynamics, and in the former they can be defined conveniently. Inside averages the pseudostreaming operators are always preceded by the hard sphere equilibrium distribution $\rho_{0} \sim W_{N} \exp [-\beta K]$, where $K$ is the total (conserved) kinetic energy. The overlap function $W_{N}=1$ for nonoverlapping configurations, and $W_{N}$ $=0$ for overlapping ones. Consequently, inside averages the pseudostreaming operators generate the correct forward and backward hard sphere dynamics. For instance, the forward time correlation function $\left\langle A e^{t L_{+}} A\right\rangle_{0}$ with $t>0$ is equal to the time-reversed one $\left\langle A e^{-t L_{-}} A\right\rangle_{0}$.

In Sec. II we need, apart from the pseudo-Liouville operator $\mathcal{L}$, also its adjoint $\tilde{\mathcal{L}}$, its conjugate $\mathcal{L}^{\epsilon}$ with $\overline{\mathcal{L}}=\widetilde{\mathcal{L}}^{\epsilon}$ according to Eq. (2.6). These operators can be expressed in binary collision operators $T_{ \pm}(i j)$ and $\bar{T}_{ \pm}(i j)$ as follows:

$$
\begin{gathered}
\mathcal{L}=\mathcal{L}_{0}+\sum_{i<j} T_{+}(i j) \equiv L_{+}, \\
\mathcal{L}^{\epsilon}=-\mathcal{L}_{0}+\sum_{i<j} T_{-}(i j) \equiv-L_{-}, \\
\widetilde{\mathcal{L}}=-\mathcal{L}_{0}+\sum_{i<j} \bar{T}_{-}(i j), \\
\overline{\mathcal{L}}=\mathcal{L}_{0}+\sum_{i<j} \bar{T}_{+}(i j),
\end{gathered}
$$

where $\mathcal{L}_{0}=\Sigma_{i} \mathbf{v}_{i} \cdot \partial / \partial \mathbf{r}_{i}$, and the binary collision operators satisfy the relations

$$
\bar{T}_{ \pm}=\widetilde{T}_{ \pm}^{\epsilon}=\widetilde{T}_{\mp},
$$

as can be verified from the definitions below. The last equality on the first and second line of Eq. (A1) are the corresponding notations used in the hard sphere kinetic theory (e.g., Refs. $[7,32])$. The binary collision operators are defined as

$$
T_{ \pm}(i j)=\sigma^{d-1} \int^{(\mp)} d \hat{\sigma}\left|\mathbf{v}_{i j} \cdot \hat{\sigma}\right| \delta\left(\mathbf{r}_{i j}-\sigma \hat{\sigma}\right)\left(b_{\sigma}-1\right),
$$




$$
\bar{T}_{ \pm}(i j)=\sigma^{d-1} \int^{(\mp)} d \hat{\sigma}\left|\mathbf{v}_{i j} \cdot \hat{\sigma}\right|\left[\delta\left(\mathbf{r}_{i j}-\sigma \hat{\sigma}\right) b_{\sigma}-\delta\left(\mathbf{r}_{i j}+\sigma \hat{\sigma}\right)\right] .
$$

The superscript $(\mp)$ denotes the constraint $\mp \mathbf{g}_{i j} \cdot \hat{\sigma}>0$, restricting the $\hat{\sigma}$ integration, respectively, to the precollision and post-collision hemisphere. The $b_{\sigma}$ operator is a substitution operator, defined as

$$
\begin{aligned}
& b_{\sigma} \mathbf{v}_{i} \equiv \mathbf{v}_{i}^{\prime}=\mathbf{v}_{i}-\left(\mathbf{v}_{i j} \cdot \hat{\sigma}\right) \hat{\sigma}, \\
& b_{\sigma} \mathbf{v}_{j} \equiv \mathbf{v}_{j}^{\prime}=\mathbf{v}_{j}+\left(\mathbf{v}_{i j} \cdot \hat{\sigma}\right) \hat{\sigma} .
\end{aligned}
$$

The definitions in Eq. (A1) imply that the pseudostreaming operator does not generate a dynamics, that is time-reversal invariant, i.e., $\mathcal{L}^{\epsilon} \neq-\mathcal{L}$. On the other hand, the arguments above imply for hard sphere systems that $\langle A B(t)\rangle_{0}$ $=\langle A(-t) B\rangle_{0}$ for $t>0$. Expressing this equality in pseudostreaming operators yields the first equality below, i.e.,

$$
\int d \Gamma \rho_{0} A e^{t \mathcal{L}} B=\int d \Gamma \rho_{0} B e^{t \mathcal{L}^{\epsilon}} A=\int d \Gamma A e^{t \overline{\mathcal{L}}^{\epsilon}} \rho_{0} B .
$$

In the last equality the adjoint of $\mathcal{L}^{\epsilon}$ operator has been introduced. The first equality is based on the properties that $\rho_{0} \exp (t \mathcal{L})=\rho_{0} \exp \left(t L_{+}\right) \quad$ with $\quad t>0, \quad$ and $\quad \exp \left(t \mathcal{L}^{\epsilon}\right) \rho_{0}$ $=\exp \left(-t L_{-}\right) \rho_{0}$ with $t>0$ generate, respectively, the exact forward and backward dynamics [30]. So by comparing the utmost left and right expressions we obtain relation (2.7) for the hard sphere streaming operators in the main text. In the body of the paper we also need the property: If $a_{i}$ $=\left\{1, \mathbf{v}_{i}, v_{i}^{2}\right\}$ is a collisional invariant, then

$$
T_{ \pm}(i j)\left(a_{i}+a_{j}\right)=0 .
$$

\section{Instantaneous transport coefficients $\boldsymbol{\eta}_{\infty}$ and $\boldsymbol{\lambda}_{\infty}$}

Consider first $\Omega_{\eta \eta}$, which is defined as the instantaneous shear viscosity $\eta_{\infty}$,

$$
k^{2} \eta_{\infty}=-\rho\left\langle a_{\mathbf{k}}^{\eta_{i}} \mid \mathcal{L} a_{\mathbf{k}}^{\eta_{i}}\right\rangle=-(\beta / V)\left\langle g_{\mathbf{k} y}^{*}\left(\mathcal{L}_{0}+\sum_{i<j} T_{+}(i j)\right) g_{\mathbf{k} y}\right\rangle_{0},
$$

where Eqs. (A1), (3.8), and (3.1) have been used for $\mathcal{L}$ and $a_{k}^{\eta_{i}}$, with $\hat{\mathbf{k}}_{\perp}^{i}$ chosen parallel to the $y$ axis. Moreover, $\mathcal{L}_{0}$ gives a vanishing contribution to Eq. (A7). Calculation of the $T_{+}(i j)$ contribution for small $k$ requires the help of Eqs. (A1), (A3), (A6), and (4.7) to show that

$$
\eta_{\infty}=-\frac{\beta m^{2}}{V}\left\langle\sum_{i<j}\left(v_{i y} r_{i x}+v_{j y} r_{j x}\right) T_{+}(i j)\left(v_{i y} r_{i x}+v_{j y} r_{j x}\right)\right\rangle_{0} .
$$

From here on the calculations for $\eta_{\infty}$ are identical to those of Eqs. (2.2)-(2.5) of Ref. [7], where the Einstein-Helfand formulas for hard spheres were taken as a starting point. We briefly sketch the different steps. For the hard sphere fluid $\eta_{\infty}$ can be expressed in terms of the hard sphere two-particle distribution function $n^{2} \varphi_{0}\left(v_{i}\right) \varphi_{0}\left(v_{j}\right) \chi$, where $\chi=g_{0}(r=\sigma+)$ is the radial distribution function at contact and $\varphi_{0}(v)$ $=(\beta m / \pi)^{d / 2} \exp \left[-\frac{1}{2} \beta m v^{2}\right]$ is the Maxwellian velocity distribution.

To carry out the subsequent integrations we change to the center-of-mass $\left\{\mathbf{R}_{i j}, \mathbf{G}_{i j}=\frac{1}{2}\left(\mathbf{v}_{i}+\mathbf{v}_{j}\right)\right\}$, and relative coordinates $\left\{\mathbf{r}_{i j}, \mathbf{v}_{i j}=\mathbf{v}_{i}-\mathbf{v}_{j}\right\}$. The result is

$$
\eta_{\infty}=-\frac{1}{8} \beta \rho^{2} \chi \int d \mathbf{r}\left\langle\left\langle g_{x} r_{y} T_{+}(i j) g_{x} r_{y}\right\rangle\right\rangle,
$$

where $g_{\alpha}$ is the relative velocity, and $\langle\langle\cdots\rangle\rangle$ denotes a Maxwellian velocity average over all particles involved. Moreover the $\mathbf{r}$ integration can be carried out because the operator $T_{+}$contains $\delta^{(d)}(\mathbf{r}-\sigma \hat{\sigma})$. The remaining integrals are $d$-dimensional generalizations of the collision integrals as appearing in the Enskog theory for hard sphere fluids (see Chap. 16.8 of Ref. [34]). Performing the $\hat{\sigma}^{-}$and $\mathbf{g}$ integrations finally yields for $\eta_{\infty}$ the result listed in Eq. (4.3) of the main text.

The calculations of the instantaneous heat conductivity $\lambda_{\infty}$ runs completely parallel to that of $\eta_{\infty}$. Starting from Eq. (3.8) we obtain

$$
\begin{aligned}
k^{2} \lambda_{\infty} & =-n \mathcal{C}_{p}\left\langle a_{\mathbf{k}}^{T} \mid \mathcal{L} a_{\mathbf{k}}^{T}\right\rangle \\
& =-\frac{\beta}{T V}\left\langle\left(e_{\mathbf{k}}^{*}-h n_{\mathbf{k}}^{*}\right) \sum_{i<j} T_{+}(i j)\left(e_{\mathbf{k}}-h n_{\mathbf{k}}\right)\right\rangle .
\end{aligned}
$$

By performing the same steps as for $\eta_{\infty}$, one sees that the terms involving the enthalpy $h n_{\mathbf{k}}$ do not contribute, and one arrives at the result

$$
\lambda_{\infty}=-\frac{\beta}{T V}\left\langle\sum_{i<j}\left(e_{i} r_{i x}+e_{j} r_{j x}\right) T_{+}(i j)\left(e_{i} r_{i x}+e_{j} r_{j x}\right)\right\rangle_{0}
$$

with $e_{i}=\frac{1}{2} m v_{i}^{2}$ for hard spheres. This expression is completely analogous to Eq. (A8), and a straightforward evaluation leads to the expression (4.3) for $\lambda_{\infty}$.
[1] J. P. Hansen and I. R. McDonald, Theory of Simple Liquids (Wiley, New York, 1986).

[2] M. P. Allen and D. J. Tildesley, Computer Simulations in Liquids (Wiley, New York, 1987).

[3] M. H. Ernst and J. R. Dorfman, J. Stat. Phys. 12, 311 (1975).
[4] For a dissident's voice, see N. G. van Kampen, Phys. Norv. 5, 278 (1971).

[5] M. H. Ernst and R. Brito, Europhys. Lett. (to be published).

[6] M. H. Ernst (in preparation).

[7] J. W. Dufty and M. H. Ernst, Mol. Phys. 102, 2123 (2004). 
[8] J. Piasecki, L. Bouquet, and J-P Hansen, J. Stat. Phys. 76, 505 (1994); Physica A 218, 125 (1995).

[9] M. H. Ernst and J. W. Dufty, Phys. Lett. A 138, 391 (1989); J. W. Dufty and M. H. Ernst, J. Phys. Chem. 93, 7015 (1989).

[10] T. Ihle and D. M. Kroll, Phys. Rev. E 67, 066705 (2003); 67, 066706 (2003).

[11] P. J. Hoogerbrugge and J. M. V. A. Koelman, Europhys. Lett. 19, 191 (1995).

[12] P. Español and P. Warren, Europhys. Lett. 56, 1676 (1997).

[13] C. A. Marsh, G. Backx, and M. H. Ernst, Phys. Rev. E 56, 1676 (1997).

[14] P. Español, Phys. Rev. E 52, 1734 (1995).

[15] M. Ripoll and M. H. Ernst, Phys. Rev. E 71, 041104 (2005).

[16] H. C. Oettinger, Beyond Equilibrium Thermodynamics (WileyInterscience, New York, 2005).

[17] S. Hess, M. Kröger, and D. Evans, Phys. Rev. E 67, 042201 (2003).

[18] V. Romero-Rochín and J. M. Rubí, Phys. Rev. E 58, 1843 (1998).

[19] J. Bonet-Avalos and A. D. Mackie, Europhys. Lett. 40, 141 (1997).

[20] P. Español, Europhys. Lett. 40, 631 (1997).

[21] P. Español and M. Revenga, Phys. Rev. E 67, 026705 (2003).

[22] M. Ripoll, M. H. Ernst, and P. Español, J. Chem. Phys. 15, 7271 (2001).

[23] P. Español and F. Vázquez, Proc. R. Soc. London, Ser. A 360, 383 (2002)

[24] P. C. Hohenberg and B. I. Halperin, Rev. Mod. Phys. 49, 435 (1977).
[25] I. Goldhirsch and T. P. C. van Noije, Phys. Rev. E 61, 3241 (2000).

[26] J. Dufty and A. Baskaran, cond-mat/0507609. (their $L=L_{+}$and their $\bar{L}=\bar{L}_{-}$in the standard notation of hard sphere kinetic theory.)

[27] H. Risken, The Fokker-Planck Equation (Springer, Berlin, 1996).

[28] C. W. Gardiner, Handbook of Stochastic Methods (SpringerVerlag, Berlin, 1983).

[29] The unconventional notation of writing the Fokker-Planck operator $\widetilde{\mathcal{L}}$ as a transpose was chosen here to unify the notation for the time dependence of dynamical variables in the form $A(t)=\exp [t \mathcal{L}] A(0)$, both for mesoscopic variables (FokkerPlanck equation), as well as for microscopic ones (Hamilton and Liouville equations).

[30] M. H. Ernst, J. R. Dorfman, W. R. Hoegy, and J. M. J. van Leeuwen, Physica (Utrecht) 45, 127 (1969).

[31] H. van Beijeren and M. H. Ernst, J. Stat. Phys. 21, 125 (1979).

[32] I. M. de Schepper, M. H. Ernst, and E. G. D. Cohen, J. Stat. Phys. 25, 321 (1981).

[33] P. Resibois and M. de Leener, Classical Kinetic Theory of Liquids (Wiley, New York, 1997)

[34] S. Chapman and T. G. Cowling, The Mathematical Theory of Non-uniform Gases, 3rd ed. (Cambridge University Press, Cambridge, 1970).

[35] H. van Beijeren and M. H. Ernst, Physica (Amsterdam) 68, 437 (1973); 70, 225 (1973). 